\title{
Right Hemisphere Activation of Joke-related Information: An Event-related Brain Potential Study
}

\author{
Seana Coulson and Ying Choon Wu
}

\begin{abstract}
Two studies tested the hypothesis that the right hemisphere engages in relatively coarse semantic coding that aids high-level language tasks such as joke comprehension. Scalprecorded event-related brain potentials (ERPs) were collected as healthy adults read probe words (CRAZY) preceded either by jokes or nonfunny controls ("Everyone had so much fun jumping into the swimming pool, we decided to put in a little water/platform"). Probes were related to the meaning of the jokes, but not the controls. In Experiment 1a, with central presentation, probes following jokes (related) elicited less negative ERPs 300-700 msec postonset (N400) than did probes following nonfunny controls (unrelated). This finding suggests related probes were primed by the jokes. In addition, unrelated probes elicited a larger anterior positivity 700900 msec than did related, as irrelevant stimuli impacted control processes invoked by task demands. In Experiment 1b,
\end{abstract}

\section{INTRODUCTION}

Over the past 2 decades, increased interest in languagerelated hemispheric asymmetries has given rise to the view that the halves of the brain process semantic information in different, perhaps complementary ways (Beeman \& Chiarello, 1998). Research with callosotomy patients indicates that in spite of its impoverished speech output, the isolated right hemisphere $(\mathrm{RH})$ has the ability to recognize words (Baynes \& Eliassen, 1998; Baynes, Tramo, \& Gazzaniga, 1992; Zaidel, 1990; Gazzaniga \& Sperry, 1967). Moreover, when left hemisphere (LH) damage causes linguistic deficits, homologous $\mathrm{RH}$ areas can sometimes be recruited to assume the compromised functions (Blasi et al., 2002; Staudt et al., 2002; Calvert et al., 2000; Buckner, Corbetta, Schatz, Raichle, \& Petersen, 1996).

Although RH lesions rarely result in severe linguistic impairment, injury to the RH has been linked to subtle semantic and pragmatic processing deficits, including difficulty understanding the meaning of idioms (Van Lancker \& Kempler, 1987), indirect requests (Stemmer, 1994; Stemmer, Giroux, \& Joanette, 1994), and jokes (Brownell, Michel, Powelson, \& Gardner, 1983). Simi-

University of California probes $(C R A Z Y)$ were preceded only by sentence-final words from jokes (water) or controls (platform). No ERP effects were observed in Experiment 1b, suggesting the N400 priming effect and the anterior positivity observed in Experiment 1a reflect semantic activations at the discourse level. To assess hemispheric differences in semantic activations, in Experiment 2, ERPs were recorded as participants read probe words presented in their left and right visual fields (LVF and RVF, respectively). Probes elicited a smaller N400 component when preceded by jokes than controls. This N400 priming effect was larger with presentation to the LVF, suggesting joke-relevant information was more active in the right hemisphere. The anterior positivity was observed with RVF but not LVF presentation, suggesting an important role for the left hemisphere in controlled retrieval in language comprehension.

larly, neuroimaging studies of healthy adults indicate that the RH is particularly important for high-level language comprehension (Bookheimer, 2002). For example, an event-related functional magnetic resonance imaging study that compared the comprehension of semantic jokes to a nonjoke baseline revealed increased activation in RH Brodmann's area 21, as well as areas 21 and 37 in the LH (Goel \& Dolan, 2001).

Other research with neurologically intact participants using the divided visual field (DVF) paradigm has revealed different patterns of priming effects with presentation to the right visual field (RVF, LH) and the left visual field (LVF, RH). Several studies have found, for example, that the RH shows facilitation to distantly related meanings, whereas the LH exhibits priming effects exclusively by close associates (Beeman, Friedman, et al., 1994; Chiarello, Burgess, Richards, \& Pollock, 1990; Burgess \& Simpson, 1988). The present studies addressed whether putative hemispheric differences in semantic activation might be relevant to joke comprehension.

\section{Joke Comprehension}

Perhaps because verbal joking is a fairly common event, we often underestimate its complexity. Joke compre- 
hension is a high-level language task that depends on our ability to flexibly apply background knowledge to make inferences both about what has been said and what is coming next (Coulson, 2000). For example, given "A thoughtful wife has pork chops ready when her husband comes home from-" most people would expect "work" to follow (Coulson \& Kutas, 2001). However, if a friend were telling you a joke and actually ended the sentence with "fishing," you would likely have no trouble understanding and chuckle politely. The word fishing here cues the listener (or reader) to make dramatic changes in her representation of the scenario. Rather than a stereotypical wife tending to the needs of her husband, we have a mischievous wife who anticipates her husband will come home empty-handed. Known as frame shifting, this semantic reanalysis process reorganizes existing information into a new frame or schema retrieved from long-term memory (Coulson \& Kutas, 2001; Coulson, 2000).

As noted above, joke-comprehension deficits have been observed in patients with RH lesions, especially when there is damage to the anterior portion of the frontal lobe (Shammi \& Stuss, 1999; Brownell et al., 1983). One attempt to link the deficits observed in RHdamaged patients to hemispheric asymmetries evident in healthy adults is Beeman's coarse coding hypothesis (Beeman \& Chiarello, 1998; Beeman, Friedman, et al., 1994). According to this hypothesis, words in the RH are represented by means of wide semantic fields, whereas words in the LH are represented via a narrow range of features relevant to the immediate discourse context. Although coarse $\mathrm{RH}$ semantic activations would predictably include contextually irrelevant information, they might nonetheless be important for the comprehension of figurative language such as that needed to understand jokes. Because jokes frequently require the integration of novel information, the reinterpretation of a word or phrase, and the reinterpretation of the scenario depicted by the preceding context, diffuse RH activation might provide additional information that makes joke processing easier. Similarly, reduced access to these diffuse semantic activations in $\mathrm{RH}$ damaged patients could result in joke-comprehension deficits.

\section{Present Study}

To test whether $\mathrm{RH}$ involvement in joke processing involves the activation of information crucial for understanding jokes, we measured event-related brain potentials (ERPs) elicited by laterally presented probe words that were preceded either by a joke or by a nonfunny control. Because all jokes turned on the last word of the sentence, control sentences were formed by replacing the sentence final word with a "straight" ending. For example, the straight ending for the joke "Everyone had so much fun diving from the tree into the swimming pool, we decided to put in a little water" was platform. Probes (such as $C R A Z Y$ ) were designed to be related to the meaning of the joke, but unrelated to the meaning of the straight control.

Note that this paradigm differs somewhat from our previous research on joke comprehension, which compared ERPs elicited by the critical words in jokes and nonfunny controls (Coulson \& Lovett, 2004; Coulson \& Kutas, 2001). Rather than measuring on-line processing of the jokes themselves, ERPs to probes index the extent to which joke-relevant information is active in the brain. In this paradigm, the jokes and the control sentences serve as "primes" that facilitate the processing of contextually relevant probes. For this reason, the amplitude of the N400 component of the ERPs was the dependent variable of interest.

In general, N400 amplitude is seen as an index of the difficulty of integrating a word into a given context: The larger the N400, the more difficult the task of lexical integration (Kutas, Federmeier, Coulson, King, \& Munte, 2000; Osterhout \& Holcomb, 1995). Because probes were related to the meaning of the jokes, but unrelated to the meaning of the control sentences, probes would be expected to elicit larger amplitude N400 when they followed control sentences than when they followed jokes. This hypothesis was tested in Experiment 1a, where participants read centrally presented probes preceded either by jokes or by straight controls. Our goal was to establish priming specifically for probes related to the discourse-level reanalysis, or frame shifting, triggered by joke-final words. To rule out N400 priming effects due to relationships between probes and the last word of each type of sentence, Experiment 1b compared ERPs elicited by centrally presented probes preceded either by the last word of the jokes or the straight controls.

Experiment 2 assessed hemispheric differences in the activation of joke-related information by recording ERPs as participants read lateralized probe words primed either by the jokes or nonfunny controls (see Figure 1). Parafoveal presentation of probe words was intended to affect the hemisphere that received the initial information from the stimulus and to increase that hemisphere's participation in stimulus processing. If hemispheric differences in semantic activation are relevant for joke comprehension, visual field (VF) of presentation might be expected to modulate ERP effects observed in Experiment $1 \mathrm{a}$.

The coarse coding hypothesis, for example, suggests that the wide semantic fields in the $\mathrm{RH}$ provide information important for understanding jokes. If this is the case, probe words related to jokes should be easier to process with LVF than RVF presentation. In the present study, greater RH activation of joke-relevant information would be indexed by larger N400 relatedness effects with presentation to the LVF than the RVF. Alternatively, similar-sized N400 relatedness effects in the two VFs would suggest either that hypothesized differences in 


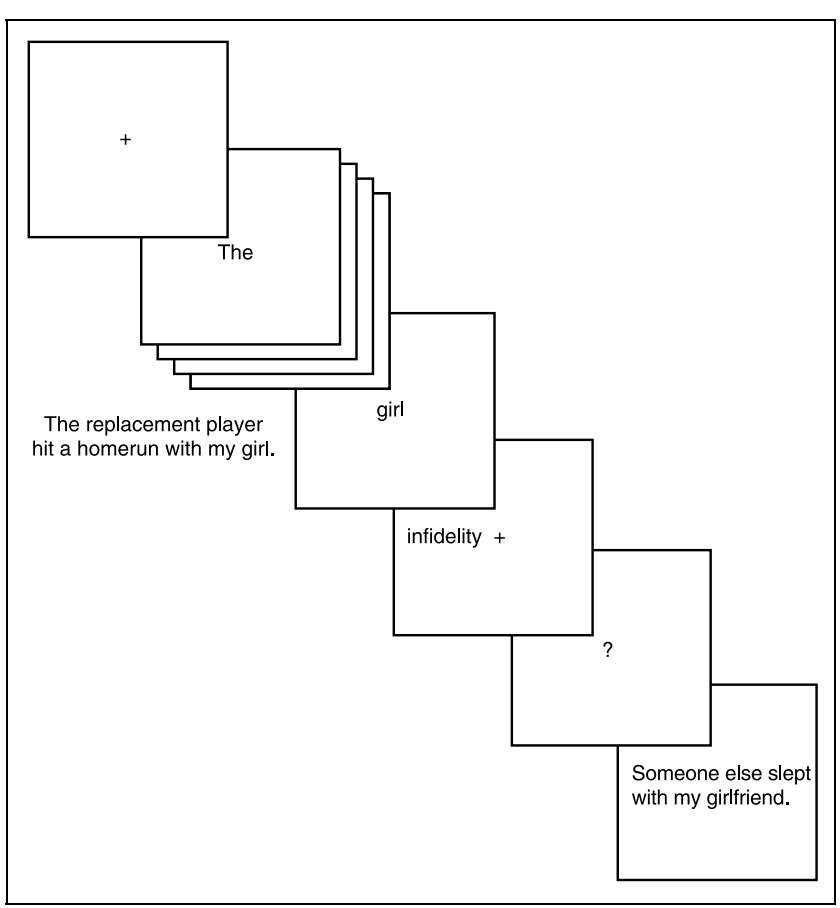

Figure 1. Sample trial.

semantic activation do not exist or that they are not relevant to joke comprehension.

\section{EXPERIMENT 1}

\section{Results}

Participants in Experiment 1 performed almost perfectly on the delayed naming task, presumably because the probe words were presented in the center of the screen. As a result, accuracy rates on the delayed naming task were not analyzed. In contrast, accuracy rates on the comprehension questions that followed the sentential stimuli in Experiment 1a were less than perfect, although participants answered most of the questions correctly $(77 \%, S D=5 \%)$. Performance on comprehension questions was the same for sentences that ended as jokes and sentences that had straight endings $(F<1)$.

ERPs to centrally presented probes following sentence and word primes can be seen in Figure 2. N400 amplitude was assessed by measuring the mean amplitude of ERPs 300-700 msec after the onset of the probe words (Van Petten, 1995). Repeated measures analysis of variance (ANOVA) with factors context (sentence/word), relatedness (related/unrelated), and electrode (28 levels) indicated a marginally significant interaction among context, relatedness, and electrode, $F(27,405)=2.19$, $p=.067$, suggesting that the size and scalp distribution of the N400 effect was not identical across conditions. Further analyses of data restricted to medial electrode sites revealed a significant interaction among context, relatedness, and hemisphere, $F(1,15)=6.26, p<.05$, reflecting a right-lateralized relatedness effect for probes preceded by sentence-but not by word-primes. A similar interaction among context, relatedness, and hemisphere was observed in the analysis of data collected from lateral electrode sites, $F(1,15)=7.06, p<.05$. Significant interactions among context, relatedness, and electrode site were also observed between 700 and $900 \mathrm{msec}$ postprobe onset, $F(27,405)=6.8, p<.001$. ERPs elicited by probes preceded by sentence primes are analyzed below under the heading Experiment 1a, and probes preceded by word primes are analyzed under the heading Experiment $1 \mathrm{~b}$.

\section{Experiment 1a: Sentence Primes}

In the sentence condition, probes following jokes (related) elicited less negative ERPs $300-700$ msec postonset than did probes following nonfunny controls (unrelated), as suggested by a reliable interaction between relatedness and electrode site, $F(27,405)=5.75$, $p<.05$. In keeping with the typical distribution of the N400 component (Kutas, Van Petten, \& Besson, 1988), the relatedness effect was largest over posterior sites [midline: Relatedness $\times$ Posteriority, $F(5,75)=5.75, p<$ .01 ; medial: Relatedness $\times$ Posteriority, $F(6,90)=5.36$, $p<.05$ ] and slightly larger over the RH [medial: Relatedness $\times$ Hemisphere, $F(1,15)=7.09, p<.05$; lateral: Relatedness $\times$ Hemisphere, $F(1,15)=6.25, p<.05]$.

In the latter part of the epoch, relatedness effects differed over anterior and posterior scalp regions in polarity. Over posterior sites, unrelated probes continued to elicit more negative ERPs than did the related

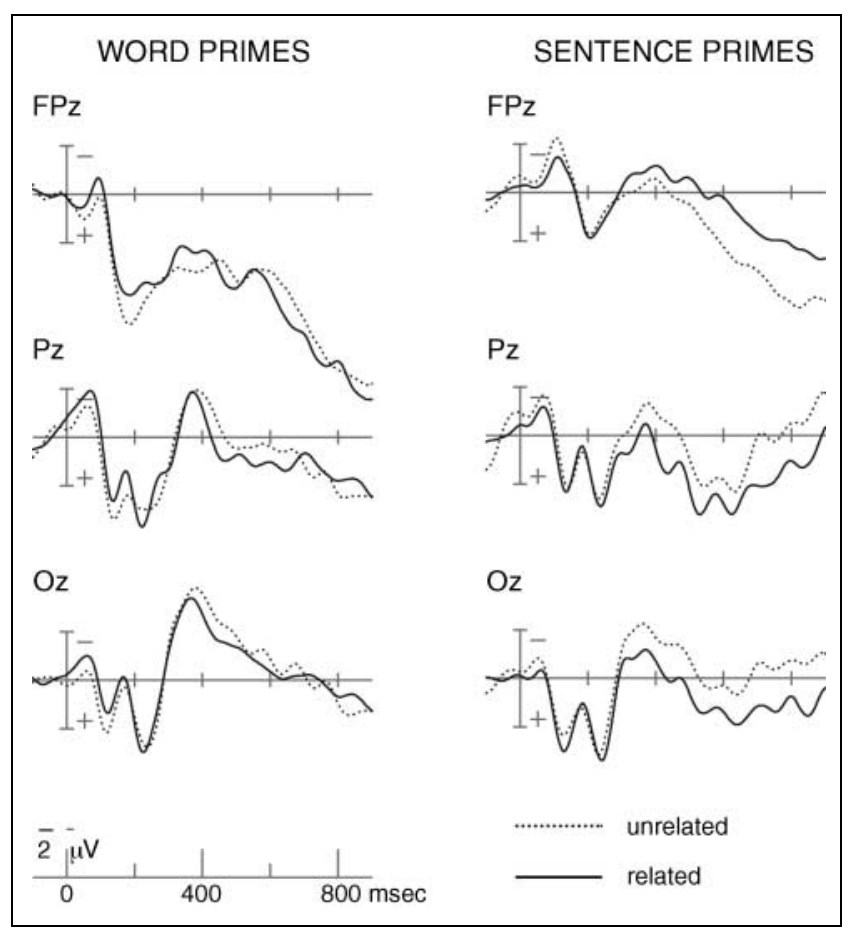

Figure 2. Relatedness effects with word and sentence primes. 
probes. Over prefrontal and frontal sites, unrelated probes elicit greater positivity beginning approximately $600 \mathrm{msec}$ postonset and continuing until the end of the epoch. Analysis of data measured $700-900 \mathrm{msec}$ postprobe onset thus revealed a reliable interaction between relatedness and electrode site, $F(27,405)=10.68$, $p<.0001$. Follow-up analyses at midline, $F(5,75)=$ $11.64, p<.0001$, and medial, $F(6,90)=14.63, p<.0001$, electrode sites revealed interactions between relatedness and posteriority, reflecting the frontal distribution of the positivity to unrelated stimuli and the posterior distribution of the negativity. Analysis also suggested an interaction between relatedness and hemisphere because of the larger negative-going effect over RH sites [medial, $F(1,15)=9.34, p<.01$; lateral, $F(1,15)=9.27$, $p<.01]$.

\section{Experiment 1b: Word Primes}

Probes that followed single-word primes elicited ERPs typical of visually presented language stimuli-namely, $\mathrm{N} 1$ and P2 visual potentials, followed by the N400. A late positive-going response was also observed beginning approximately $600 \mathrm{msec}$ postonset over frontal and prefrontal sites. However, the relatedness factor did not affect the amplitude of the ERPs measured 300700 msec [relatedness, $F(1,15)=2.36, n s$; Relatedness $\times$ Electrode, $F(27,405)<1$ ] or 700-900 msec [relatedness, $F(1,15)<1$; Relatedness $\times$ Electrode, $F(27,405)<1$ ] postonset. This null result is not surprising, given that relatedness judgments depend upon the relationship between probe words and the meaning of the sentences (although caution is always warranted in the interpretation of a null result).

\section{Discussion}

The goal of Experiment 1 was to show that our jokerelated probes were primed more by the jokes than by the nonfunny controls, and further, that this priming was because of sentence- or discourse-level information rather than lexical relationships between the probes and sentence-final words. Nonfunny controls were designed to prompt essentially the same discourse-level representation as jokes with the exception of information activated by the reanalysis needed to get the joke. Consequently, the same probe words could be considered related to jokes, but unrelated to controls. N400 relatedness effects can thus be seen as indicating that jokes activated information important for comprehending the humor.

As predicted, unrelated probes elicited larger amplitude N400 components than did related probes in Experiment 1a (sentence primes), but not in Experiment $1 \mathrm{~b}$ (word primes). Similarly, unrelated probe words elicited a larger positivity $700-900 \mathrm{msec}$ over anterior scalp sites in Experiment 1a, but not in Experiment 1b. Because the joke and the control sentences in Experiment 1a were identical until the final word, and those words were used as the primes in Experiment 1b, ERP effects observed in Experiment 1a but not in Experiment $1 \mathrm{~b}$ reflect sensitivity to sentence- and/or discourse-level processing, rather than to lexical associations. Related probes elicited lower amplitude N400 because they were primed by the meaning of the jokes, but not by the nonfunny control stimuli. Further, we speculate that the unrelated probes elicited a larger frontal positivity because they were not relevant to the meaning of preceding sentence primes.

Experiment 2 tested whether relatedness effects were modulated by VF of presentation. As in Experiment 1, ERPs were recorded as healthy adults read sentence primes, presented one word at a time in the center of a computer monitor. Shortly after the offset of the sentence final word, a probe was briefly flashed in either the LVF or the RVF. Participants named the probe when cued by a prompt that appeared 2 sec after its onset and answered true/false comprehension questions about the sentence primes (see Figure 1). If hemispheric differences in semantic activation affect joke comprehension, the VF manipulation would be expected to modulate the size of the $\mathrm{N} 400$ relatedness effect.

\section{EXPERIMENT 2}

\section{Results}

Volunteers correctly named an average of $71 \%$ ( $S D=$ $2 \%$ ) of the probe words. Probes presented to the RVF $(80 \%, S D=1 \%)$ were named more accurately than those presented to the $\operatorname{LVF}(62 \%, S D=1 \%)$, as suggested by a VF effect in the analysis, $F(1,15)=114$, $p<.0001$. This outcome may be attributed to superior reading abilities in the $\mathrm{LH}$ and suggests the lateralized presentation of the stimuli resulted in the increased participation of the hemisphere opposite the VF of presentation. There was no main effect of relatedness on accuracy, nor was there a reliable interaction between $\mathrm{VF}$ and relatedness $(F ' s<1)$.

Accuracy scores on the comprehension questions that followed the probes suggested that participants understood most of the sentences. VF of probe presentation did not affect accuracy scores $(F<1)$, but analysis suggested a nonsignificant trend toward better comprehension of sentences with joke $(84 \%, S D=2 \%)$ than straight $(79 \%, S D=2 \%)$ endings, $F(1,15)=3.7$, $p=.08$.

Besides the RVF advantage that participants displayed on the delayed naming task, the DVF presentation also affected the amplitude and latency of the N1 component, a well-studied ERP component implicated in highlevel visual processing (Hillyard \& Anllo-Vento, 1998). To assess the amplitude of the N1, we measured the mean 
amplitude of ERPs elicited from 75 to 175 msec after the onset of lateralized stimuli at four electrode sites where $\mathrm{N} 1$ is known to be prominent (T5, T6, O1, and O2) (Federmeier \& Kutas, 1999, 2002). The N1 was larger over RH electrode sites with LVF presentation and larger over LH electrode sites with RVF presentation [VF $\times$ Hemisphere, $F(1,15)=12.85, p<.05]$. As evident in Figure 3, this N1 reversal was more pronounced at temporal than occipital electrode sites [VF $\times$ Hemisphere $\times$ Posteriority, $F(1,15)=8.21, p<.05]$. The larger amplitude of the N1 over the hemisphere contralateral to the VF of presentation suggests that DVF presentation led to the increased participation of the opposite hemisphere.

\section{N400 component}

The N400 component was assessed by measuring the mean amplitude of ERPs elicited between 300 and 700 msec poststimulus. Repeated measures ANOVA revealed a main effect of relatedness, $F(1,15)=6.64$, $p<.05$, qualified by an interaction among relatedness, $\mathrm{VF}$, and electrode site, $F(28,420)=2.17, p<.05$. Unrelated probes elicited more negative ERPs than did related probes, especially over posterior electrode sites where N400 tends to be largest. The three-way interaction results because this relatedness effect was larger and more broadly distributed with presentation to the LVF than the RVF (see Figures 4 and 5). This interaction motivated separate analyses of data associated with presentation to each VF.

Presentation to the LVF resulted in more negative ERPs to unrelated than related probes from 300 to 700 msec postonset, as indicated by main effects of relatedness at the midline, $F(1,15)=11.88, p<.01$, medial, $F(1,15)=11.17, p<.01$, and lateral, $F(1,15)=$ $5.03, p<.05$, electrode sites. Consistent with the distribution of the N400 component (Kutas, Federmeier, et al., 2000), the negativity was largest over central and parietal electrode sites [midline: Relatedness $\times$ Posteriority, $F(6,90)=17.45, p<.01$; medial: Relatedness $\times$ Pos- teriority, $F(6,90)=15.5, p<.01]$ and was slightly larger over RH electrode sites [medial: Relatedness $\times$ Hemisphere, $F(1,15)=3.38, p=.08$; lateral, $F(1,15)=5.34$, $p<.05]$.

With RVF presentation, unrelated stimuli elicited more negative ERPs 300-700 msec than related stimuli over posterior electrode sites and less negative ERPs than related stimuli over frontal sites. The analyses revealed no main effect of relatedness, but rather reliable interactions between relatedness and the factor indexing the location of electrode sites along the anterior posterior axis [midline, $F(6,90)=18.23, p<.0001$; medial, $F(6,90)=11.33, p<.001$; lateral, $F(3,45)=11.81, p<$ $01]$. Consistent with the scalp distribution of the $\mathrm{N} 400$, the negative-going relatedness effect was largest over posterior RH electrode sites [medial: Relatedness $\times$ Hemisphere, $F(1,15)=8.03, p<.05$; lateral: Relatedness $\times$ Hemisphere, $F(1,15)=5.74, p<.05$; lateral: Relatedness $\times$ Hemisphere $\times$ Posteriority, $F(3,45)=$ $5.88, p<.01]$. However, the presence of less positive ERPs to unrelated than related probes over frontal electrode sites differs from the typical N400 effect.

\section{Analysis from 700 to $900 \mathrm{msec}$}

An omnibus ANOVA of mean amplitude of ERPs measured 700-900 msec poststimulus onset revealed an interaction between relatedness and electrode site, $F(28,420)=9.37, p<.0001$, as well as a trend toward an interaction between relatedness and $\mathrm{VF}, F(1,15)=$ $3.76, p=.07$. As can be seen in Figure 4 , LVF presentation resulted in a continuation of the $\mathrm{N} 400$ relatedness effect, as ERPs to unrelated probes were more negative than were ERPs to related ones [midline, $F(1,15)=4.82$, $p<.05$; medial, $F(1,15)=4.9, p<.05]$. As in the previous time window, the negativity was largest over posterior scalp regions [midline: Relatedness $\times$ Posteriority, $F(6,90)=5.6, p<.01$; medial: Relatedness $\times$ Posteriority, $F(6,90)=2.85, p=.07]$. In contrast, RVF presentation resulted in a positive-going response to unrelated probes over prefrontal and frontal electrode

Figure 3. N1 visual potentials.

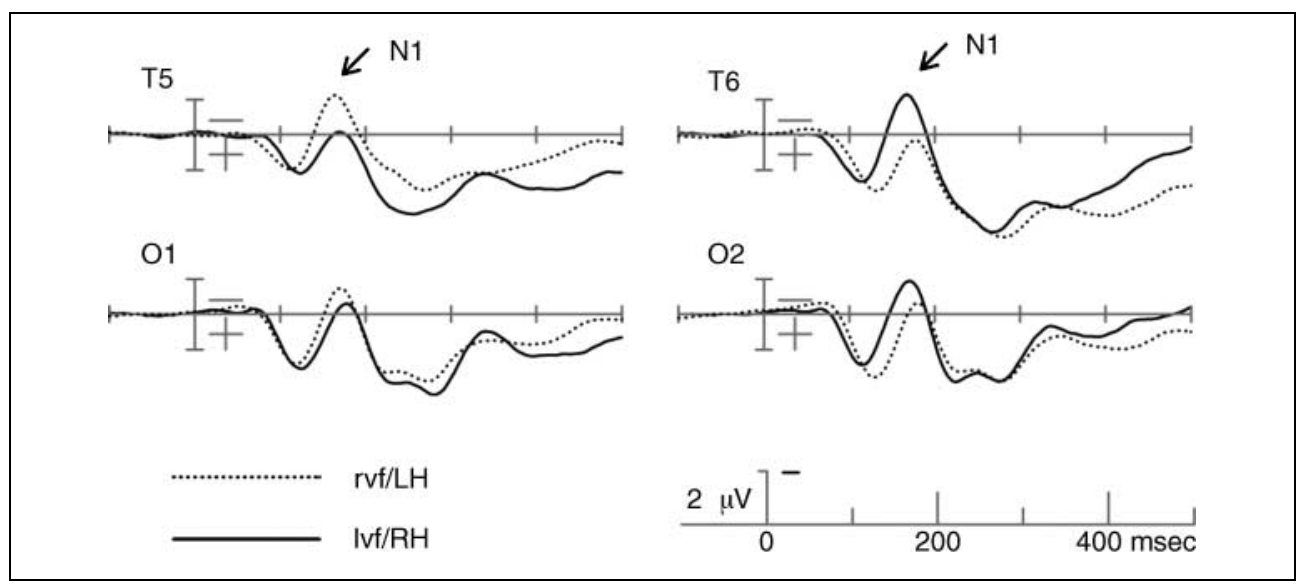




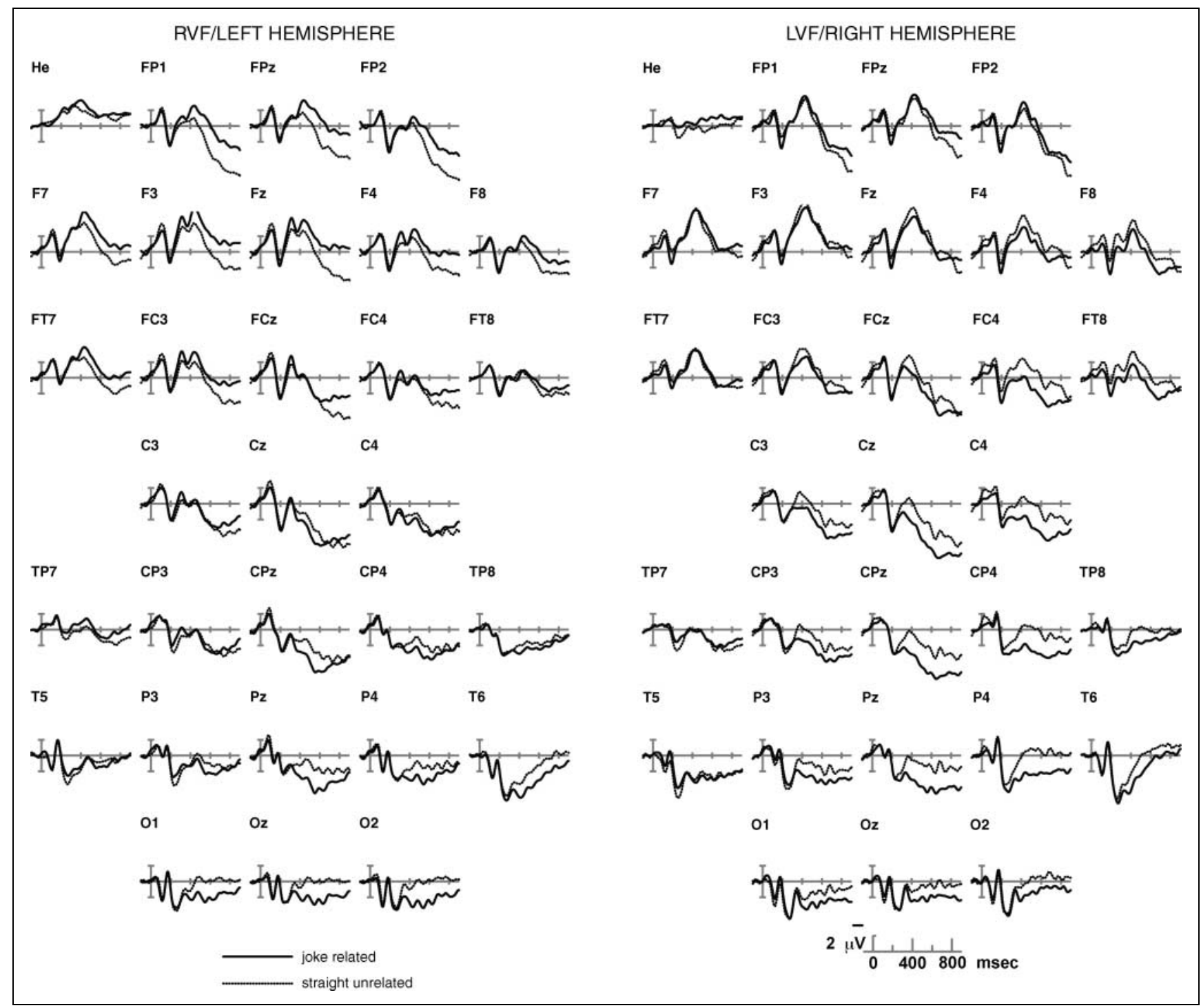

Figure 4. Relatedness effect in RVF and LVF.

sites and a small negative-going response over more posterior sites [midline: Relatedness $\times$ Posteriority, $F(6,90)=18.54, p<.001 ;$ medial: Relatedness $\times$ Posteriority, $F(6,90)=12.47, p<.001$; lateral: Relatedness $\times$ Posteriority, $F(3,45)=10.78, p<.01]$.

\section{Discussion}

As in Experiment 1, related probes in both VFs elicited less negative ERPs than unrelated 300-700 msec postonset. This finding suggests semantic activations in both hemispheres supported the priming of joke-related information. Moreover, this N400 priming effect was larger with presentation to the LVF than the RVF, suggesting joke-relevant information was more active in the RH. Larger LVF effects result from both relatively more positive ERPs to related probes and more negative ERPs to unrelated probes.
With RVF presentation, unrelated probes also elicited more positive ERPs over frontal electrode sites in the latter part of the epoch. This effect was largely absent with LVF presentation. Although a hint of a positivegoing response is evident in the waveforms collected from prefrontal sites (see Figure 4), the LVF positivity is much smaller and less broadly distributed than observed with RVF presentation; it appears to begin later and end earlier than with RVF presentation and was not statistically significant. The functional significance of the anterior positivity is discussed more extensively below.

\section{GENERAL DISCUSSION}

The present study was aimed at evaluating whether hemispheric differences in semantic activation affect joke comprehension. In Experiment 1a, ERPs were re- 
Figure 5. Relatedness difference waves (unrelated minus related).

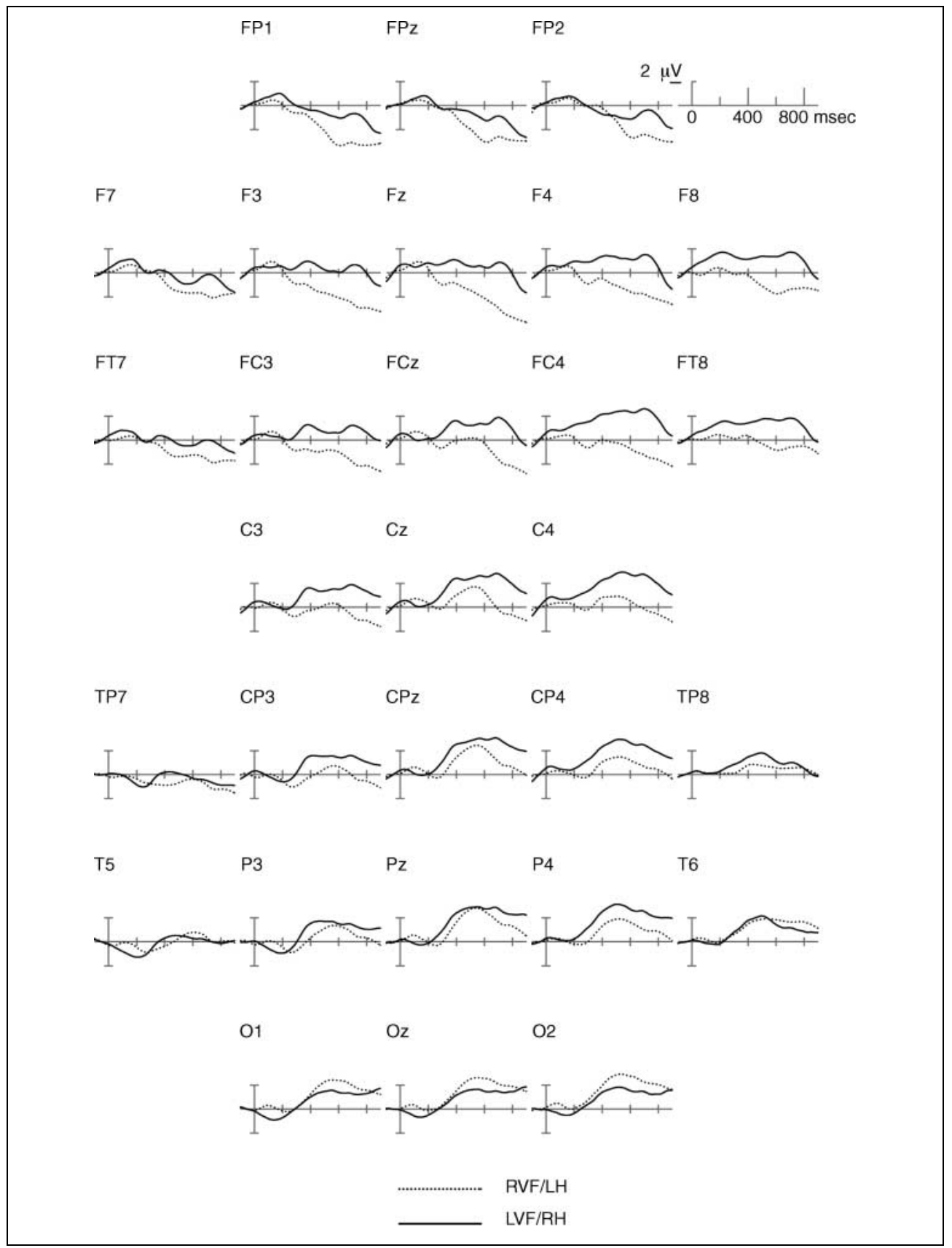

corded as healthy adults read centrally presented probe words (e.g., CRAZY) preceded either by a joke (e.g., "Everyone had so much fun diving from the tree into the swimming pool, we decided to put in a little water,") or by a control sentence with a straight ending (e.g., "Everyone ... a little platform."). Probes were related to the meaning of the jokes but unrelated to the meaning of the control sentences.

Relative to the unrelated probes, related probes elicited a smaller N400, suggesting semantic integration of joke-relevant information was easier after the jokes. Further, relative to related probes, unrelated probes elicited a more positive-going ERP response over frontal electrode sites, the anterior positivity. As discussed below, we suggest that the anterior positivity indexes the brain's recognition (either implicit or explicit) of the contextual irrelevance of the unrelated probes.

In Experiment 1b, ERPs were recorded as participants read centrally presented probe words from Experiment 1a. The primes, however, were single words-sentencefinal words from the joke stimuli and their controls from Experiment 1a (e.g., water-CRAZY, platform-CRAZY), as these were the single word in the sentence contrasts that differed. In this experiment, "unrelated" probes (primed by the last word of the control sentences) elicited similar amplitude N400 as the "related" probes (primed by the last word of the jokes). The anterior positivity was also unaffected by "relatedness" (prime 
type) in this experiment. Thus, priming of joke-relevant information observed in Experiment 1a depends on a message-level interpretation of the sentential primes rather than lexical priming between sentence-final words in the joke and the joke-related probes.

In Experiment 2, probe words primed either by jokes (related) or controls (unrelated) were presented in participants' left and right VFs. Consistent with predictions of the coarse coding hypothesis, LVF presentation did indeed result in larger $\mathrm{N} 400$ relatedness effects in Experiment 2 (see Figure 5). Over posterior sites, ERPs to related probes were more positive with LVF than RVF presentation, and ERPs to unrelated probes were more negative, suggesting that joke-related information was more active in the RH than the $\mathrm{LH}$ in this time window.

Unfortunately, this interpretation is clouded by the presence of a partially overlapping anterior positivity that occurred with presentation to the RVF but not the LVF. Summation of a positive- and a negative-going response could easily result in the apparent attenuation of the RVF N400 effect. However, the presence of an overlapping anterior positivity in the RVF condition cannot explain our finding that ERPs to related probes measured 300-700 msec postonset over posterior midline electrodes were more positive with presentation to the LVF than RVF. This latter result can be interpreted as indicating the $\mathrm{RH}$ found the related probes to be less surprising than did the LH, assuming more positive ERPs in the N400 latency window index ease of lexical integration. Of course, inferences from an amplitude comparison between VFs (even in the within-subjects design employed here) are open to question.

Taken at face value, however, the larger $\mathrm{N} 400$ relatedness effect with LVF presentation suggests that jokerelevant information was more active in the RH. This result is consistent with other research in the DVF priming paradigm that indicates semantic activations in the $\mathrm{RH}$ are important for high-level language comprehension tasks. For example, solution-relevant information for linguistic insight problems is more active in the RH than the LH (Beeman \& Bowden, 2000) Like jokes, insight problems involve the reinterpretation of deliberately ambiguous information and are accompanied by a sudden feeling of "getting it." Further, in a study of inferences drawn during story comprehension, probe words related to predictive inferences (e.g., LAUNCH after "The shuttle sat on the ground in the distance") showed priming only with presentation to the LVF, suggesting RH semantic activation supports predictive inferencing in story comprehension (Beeman, Bowden, \& Gernsbacher, 2000).

\section{Right Hemisphere Semantic Activations}

Our finding that joke-related probe words elicited different ERP effects with presentation to the left and right VFs suggests hemispheric differences in semantic activa- tion are indeed relevant to joke comprehension. Further, the larger N400 priming effect with LVF presentation is consistent with the coarse coding hypothesis that RH semantic activation is less specific than that in the LH. However, this leaves open the precise nature of the differences between semantic activation in the two hemispheres.

The most straightforward interpretation of the coarse coding hypothesis-sensitivity to associative relationships-appears not to be supported by existing data. If the RH activates a wider array of information, somewhat irrespective of its contextual relevance, one would expect it to be less affected than the LH by differences in associative strength between words. However, in a DVF priming study that compared how associative strength affected the size of priming effects, Coney (2002) found a similar function with both LVF and RVF presentation.

Rather than semantic distance, per se, we suggest that such hemispheric differences reflect RH coding of thematic and relational information. RH damaged patients have greater difficulty than those with LH damage at recalling the theme of stories they have read (Hough, 1990). Further, neuroimaging research with healthy adults also suggests the importance of RH temporal lobe activity in processing thematic information. In a story comprehension task using materials modeled after the classic study by Bransford and Johnson, St. George, Kutas, Martinez, and Sereno (1999) report greater RH temporal lobe activation when thematic information was not provided for ambiguous paragraphs than when thematic information was provided by the inclusion of titles for the paragraphs.

One chief source of evidence for the coarse coding hypothesis comes from an experiment in which centrally presented trios of words served as "summation primes" for a weakly related lateralized target (Beeman, Friedman, et al., 1994). For example, cry, foot, glass served as summation primes for the target CUT and as direct primes for the target $L A U G H$, which was semantically related to one of the prime words. Accuracies on a naming task indicated that, relative to unrelated items, both hemispheres benefited from both sorts of primes. However, there was a RVF advantage for the direct primes, and an LVF advantage for summation primes. Beeman et al. argue that these findings suggest the RH benefits from the summed activation of shared features within a set of semantic fields. Note, however, that the features of foot and glass do not intersect. Rather, glass can be construed as an instrument that induces a change of state in a patient, such as a foot. These considerations point to the possibility that the RH "appreciates" the relational structure that links summation primes.

Summation priming may result because the RH preferentially activates stored knowledge about typical situations when glass, feet, and crying might co-occur, 
whereas the LH is more sensitive to syntactic cues that overtly specify the same types of complex relations. Like Beeman's summation triads, jokes require the listener to access nonovertly encoded information to understand the connection between one's initial interpretation and the construal implied by the joke's punch line. For example, in the joke "The replacement player hit a home run with my girl," the reader must reinterpret information about a baseball game by accessing information about romance. Thus, hemispheric differences in semantic activation might explain hemispheric differences in joke comprehension.

\section{Neural Basis of Semantic Activation Differences}

These findings raise the issue of the anatomical and physiological causes of observed hemispheric differences. Previously, hemispheric differences in semantic activation have been linked to greater connectivity in the RH (Beeman \& Chiarello, 1998). Research indicates a greater extent of dendritic branching in language areas of the RH and LH (Jacobs, Batal, et al., 1993; Jacobs, Schall, Scheibel, 1993) and a higher density of interneurons (Hutsler \& Gazzaniga, 1995). Similarly, there is more interconnectivity in the $\mathrm{RH}$, both at the level of individual neurons and at the level of cortical columns (Hutsler \& Galuske, 2003). The wider spacing of cortical columns in posterior language regions in the $\mathrm{LH}$ could be conducive to coding for more distinct features, whereas RH cortical columns are less functionally differentiated (Hutsler \& Galuske, 2003; Galuske, Schlote, Bratzke, \& Singer, 2000).

Asymmetries in cortical microcircuitry could thus give rise to less distinctive coding in $\mathrm{RH}$ posterior language regions, and might make them more suited for coding abstract, relational features. Indeed, the very definition of an abstract feature is a property shared by superficially different exemplars. The concept fruit, for example, is more abstract than apple, and different exemplars of apples share more perceptual features than do different exemplars of fruits. Similarly, understanding the relational concept taller involves abstracting over multiple comparisons of people, buildings, and other objects of varying sizes and shapes.

Alternatively, differences in semantic activation might be related to hemispheric differences in attentional bias, as studies of visuospatial processing suggest the $\mathrm{RH}$ is superior in tasks that require the direction of attention to global (whole object) aspects of stimuli, whereas the $\mathrm{LH}$ is better at attending to local (object features) aspects (Yamaguchi, Yamagata, \& Kobayashi, 2000; Fink et al., 1996; Van Kleek, 1989; Delis, Robertson, \& Efron, 1986). Such biases might affect the way that information is encoded into memory, which in turn, might affect the way it is retrieved during inferential aspects of language comprehension.

\section{Anterior Positivity}

Perhaps the most striking VF effect in the present study was not the N400 priming effect, but the anterior positivity. Compared with related probes, unrelated probes elicited more positive ERPs over frontal recording sites. One possible interpretation of this effect is that it stems from the demands of the delayed naming task. Note however, that the absence of relatedness effects in Experiment $1 \mathrm{~b}$ suggests the anterior positivity reflects sensitivity to context rather than the naming task per se. Participants named the same words in Experiments 1a and $1 \mathrm{~b}$ (and in related and unrelated conditions), but the unrelated probes were only viewed as unrelated when they followed the sentences in 1a.

With central presentation, the anterior positivity was larger over LH electrode sites, consistent with a generator in LH prefrontal cortex. An LH generator is also supported by the fact that the positive-going relatedness effect begins earlier with RVF than central presentation (400 vs. 600 msec postonset) and is absent with LVF presentation. An LH generator for this effect is consistent with neuroimaging research that reveals left inferior frontal activation in tasks targeting long-term memory encoding and retrieval, working memory, and speech generation (Buckner, 2003; Duncan \& Owen, 2000). More relevantly, areas in the anterior portion of the left inferior prefrontal cortex have been shown to increase in activation as a function of semantic distance between words (Roskies, Fiez, Balota, Raichle, \& Petersen, 2001; Wagner, Pare-Blagoev, Clark, \& Poldrack, 2001). Relatedness effects on the anterior positivity may thus reflect activity in brain regions associated with selective retrieval of information from semantic memory.

On this account, contextually unrelated probes place more demands on control processes that gate the retrieval of relevant information in language comprehension. Consistent with this interpretation, DVF priming studies indicate the $\mathrm{RH}$ is less effective than the $\mathrm{LH}$ at suppressing contextually irrelevant information (Faust \& Gernsbacher, 1996; Burgess \& Simpson, 1988). Given the role of the left inferior prefrontal cortex in controlled retrieval processes (Buckner, 2003; Wagner et al., 2001), the exclusively RVF elicitation of the anterior positivity may reflect an important role for the $\mathrm{LH}$ in the selection of relevant and the suppression of irrelevant information (Beeman, Bowden, \& Gernsbacher, 2000). Consequently, whereas joke-relevant information might be more active in the $\mathrm{RH}$, LH selection processes may be required for the full appreciation of a joke.

\section{METHODS}

\section{Experiment 1}

\section{Participants}

Sixteen adults from the University of California-San Diego (UCSD) community (8 men), aged 18-36, were 
either paid $\$ 24$ or awarded credit for cognitive science or psychology course requirements in exchange for their participation. All were healthy, right-handed, monolingual English speakers. Handedness was assessed via the Edinburgh Inventory (Oldfield, 1971). Average laterality quotient was +71 .

\section{Materials}

Experimental stimuli were constructed from jokes found in published anthologies and deemed by the experimenters to require frame shifting for their comprehension. In all jokes, the "punch-word" was the last word of the sentence. Jokes that fit these criteria were normed in an off-line cloze (sentence completion) task administered to a minimum of 45 people. Results of the cloze task enabled us to choose nonfunny "straight" endings for the sentences that matched joke endings for length (6.8 characters), frequency (46.8 per million), and cloze probability (2.5\%). Straight endings were chosen to be consistent with the frame or schema evoked by the first part of the sentence, in contrast to joke endings that prompted frame shifting. The sentence constraint of experimental stimuli-operationalized as the cloze probability of the most common response for each item on our cloze task-varied from $7 \%$ to $96 \%$ and averaged $35 \%$.

Probe words were selected so as to be related to the humorous inferences prompted by the jokes and unrelated to the thematic content of the straight controls. Relatedness between probe words and the two types of sentence primes was assessed in a norming study in which 66 UCSD students (34 volunteers for List 1 and 32 for List 2) rated the degree of relatedness between each probe word and one of its sentence primes. On a scale from 1 to 7 , where 1 was unrelated and 7 was bighly related, probes following jokes were rated 5.4 $(S E=.36)$, whereas probes following straights were rated $2.9(S E=.63)$. Although sentences with joke and straight endings differed by only one word, probes were judged to be related to the meaning of the jokes and unrelated to the straight sentences.

Experimental stimuli consisted of 140 probes, 140 jokes, and 140 straight control sentences. Because probes were always related to jokes and unrelated to straights, materials also included a total of 240 filler probes, primed by either 120 jokes or 120 straight controls. Filler probes matched experimental probes in word length and word frequency. Filler probes following jokes were judged as being unrelated in the norming study described above (rated 2 on our 7-point scale), whereas fillers following straights were judged as related (rated 5.7 on the same scale).

In Experiment 1a (sentences), each participant read 70 experimental probes, half of which were preceded by jokes, half of which were preceded by straights, as well as 60 filler probes, half preceded by jokes and half by straights. The same people participated in Experi- ment $1 b$ (words), in which each participant read 70 experimental probes, half preceded by a sentence-final word taken from a joke and half by a sentence-final word taken from a straight control sentence. Sixty filler probes were also included, half preceded by a sentencefinal word taken from a filler joke and half by a sentencefinal word taken from a filler straight. Four lists were constructed so that no individual participant saw more than one version of any prime, but manipulations were perfectly counterbalanced across participants. Trials with sentence primes (Experiment 1a) were blocked, as were trials with single-word primes (Experiment 1b). Sentence blocks and word blocks were interleaved.

\section{Stimulus Presentation}

All trials in Experiment 1a began with a warning cross presented for $1000 \mathrm{msec}$. Sentence primes were shown one word at a time (200-msec duration and $300 \mathrm{msec}$ interstimulus interval) in the center of a 19-in. color monitor. Probes appeared $800 \mathrm{msec}$ after the offset of the sentence final word and were shown for $200 \mathrm{msec}$. Two thousand eight hundred milliseconds after the presentation of the probe word, a blue question mark appeared in the center of the screen for $3 \mathrm{sec}$ and served as the naming prompt. Immediately following the offset of the naming prompt, a comprehension question appeared on the screen (in full) for a total of $4 \mathrm{sec}$. Comprehension questions were either true or false statements about preceding sentence primes.

Trials in Experiment $1 \mathrm{~b}$ also began with a warning cross presented for 1000 msec. Prime words were presented for $200 \mathrm{msec}$. Probes appeared $800 \mathrm{msec}$ after the offset of prime, and were shown for $200 \mathrm{msec}$. Then, 2800 msec after the presentation of the probe word, a blue question mark appeared in the center of the screen for $3 \mathrm{sec}$ and served as the naming prompt.

\section{Procedure}

Participants were seated in a comfortable chair at a distance of $37 \mathrm{in}$. from the monitor. In Experiment 1a, they were told that they would be reading sentences one word at a time in the center of the screen and were instructed to focus their eyes at all times on a fixation point just below the centrally presented words. After the probe word appeared, they were to read it silently without moving their eyes and then say it aloud when the blue question mark appeared or to say "no," if they had been unable to read it. When the comprehension probe appeared, they were to press "yes" if it supported the preceding sentence or "no" if it contradicted it. (Response hand was counterbalanced across participants.) In Experiment 1b, they were told that they would be reading two words in a row and they should read the second word out loud when the blue question 
mark appeared or to say "no" if they had been unable to read it. There were no comprehension questions in this part of the experiment.

\section{Electroencephalogram Recording}

Electroencephalogram (EEG), sampled at $250 \mathrm{~Hz}$, was collected from 29 tin electrodes arranged in an expanded version of the 10-20 system (Nuwer et al., 1998), referenced to the left mastoid. ERPs were rereferenced off-line to an average of the signal collected from the left and right mastoid electrodes. Blinks and eye movements were monitored via an electrode beneath the right eye and one electrode at each of the outer canthi (the electrooculogram, EOG). Average artifact rejection rate in Experiment 1a (sentence primes) was $12 \%(S D=8 \%)$. Average artifact rejection rate in Experiment 1a (word primes) was $19 \%(S D=17 \%)$. The EEG and EOG were recorded and amplified with a set of 32 bioamplifiers from SA Instruments (San Diego, CA), with half-amplitude cut-offs at 0.01 and $40 \mathrm{~Hz}$ and digitized on a PC. Informed consent was obtained, and all procedures conformed to ethical requirements of the University of California-San Diego.

\section{Data Analysis}

Mean accuracy on the comprehension questions was assessed, with repeated measures ANOVA with relatedness as the sole factor. Because nearly every probe on the naming task was named correctly, naming accuracy was not analyzed. Mean amplitude of ERPs was measured 300-700 and 700-900 msec postprobe onset. These values were initially subjected to repeated measures ANOVA with factors context (sentence/word), relatedness (related/unrelated), and 28 levels of electrode site. In individual analyses of Experiment 1a and $1 \mathrm{~b}$, respectively, the context factor was not included. Reliable interactions between the experimental variable and electrode site were followed up with three sorts of analyses: midline (using channels $\mathrm{FPz}, \mathrm{FCz}, \mathrm{Cz}, \mathrm{CPz}, \mathrm{Pz}$, and $\mathrm{Oz}$ ), medial (using channels FP1, F3, FC3, C3, CP3, $\mathrm{P} 3, \mathrm{O} 1$, and their LH counterparts), and lateral (F7, FT7, TP7, T5, and their LH counterparts). Where appropriate, the Huhyn-Feldt correction (Huynh \& Feldt, 1978) has been applied. We report corrected $p$ values, but the original degrees of freedom have been maintained for clarity.

\section{Experiment 2}

\section{Participants}

Sixteen adults from the UCSD community (8 men), aged $18-25$, were either paid US $\$ 24$ or awarded credit hours for course requirements in exchange for their participation in the study. All were right-handed, mono- lingual English speakers with normal visual acuity and no history of neurological disorder. Handedness was assessed via the Edinburgh Inventory (Oldfield, 1971). Participants' average laterality quotient was +75 .

\section{Materials}

Materials were the same as the sentences used in Experiment 1a. Each participant read 140 experimental probes, half of which were preceded by jokes and half by straights, along with 120 filler probes, half preceded by jokes and half by straights. In each condition (experimental related and unrelated, filler-related and -unrelated), half of the probes appeared in the participant's LVF and half in her right. Four lists were constructed so that while all manipulations were counterbalanced across participants, no individual participant saw more than one version of any prime or probe.

\section{Stimulus Presentation and Procedure}

Stimulus presentation was identical to that employed in Experiment 1a, except that probe words were not presented centrally. LVF probes were presented so that their rightmost character was $2^{\circ}$ of visual angle to the left of the center of the monitor. RVF probes were presented so that their leftmost character was $2^{\circ}$ of visual angle to the right of the center of the monitor. Simultaneous with the onset of the lateralized probe, a fixation cross appeared in participants' center of gaze. As in Experiment 1, the session began with a brief practice block. In Experiment 2, participants were asked to repeat the practice block until the experimenter was satisfied that they were able to comply with task demands (refrain from horizontal eye movements during probe presentation, wait until prompted by the blue question mark to name the probe, and answer the comprehension questions with a button-press). Experimenters monitored participants' eye movements on-line via the EOG. When participants moved their eyes during probe presentation, data collection was paused and the experimenter reexplained the instructions.

\section{Electroencephalogram Recording}

EEG recording parameters were identical to Experiment 1 . The average artifact rejection rate was $15 \%$ $(S D=14 \%)$.

\section{Data Analysis}

Mean accuracy on the naming task (scored in terms of percent correct) was assessed with repeated measures ANOVA with factors VF (LVF/RVF) and relatedness (related/unrelated). Accuracy on comprehension probes (also scored in terms of percent correct) was subjected to a similar analysis. Mean amplitude of ERPs was 
measured 300-700 and 700-900 msec postprobe onset. These values were initially subjected to repeated measures ANOVA with factors VF (LVF/RVF), relatedness (related/unrelated), and 29 levels of electrode site. Reliable interactions between either of the experimental variables and electrode site were followed up with three sorts of analyses: midline (using channels FPz, Fz, FCz, $\mathrm{Cz}, \mathrm{CPz}, \mathrm{Pz}$, and $\mathrm{Oz}$ ), medial (using channels FP1, F3, FC3, C3, CP3, P3, O1, and their LH counterparts), and lateral (F7, FT7, TP7, T5, and their LH counterparts). In these analyses, the posteriority factor indexes the position of electrodes along the anterior/posterior axis of the scalp. Where appropriate (i.e., medial and lateral analyses), the hemisphere factor indexes the electrodes' location on the participant's head (over the LH or RH). In the analyses described above, the term hemisphere always refers to electrode location and never to the VF factor. Other aspects of the analyses were the same as in Experiment 1.

Reprint requests should be sent to Seana Coulson, Cognitive Science Department, 0515, 9500 Gilman Drive, La Jolla, CA 92093-0515, or via e-mail: coulson@cogsci.ucsd.edu.

\section{REFERENCES}

Baynes, K., \& Eliassen, J. C. (1998). The visual lexicon: Its access and organization in commissurotomy patients. In M. Beeman \& C. Chiarello (Eds.), Right hemisphere language comprebension (pp. 79-104). Mahwah, NJ: Erlbaum.

Baynes, K., Tramo, M. J., \& Gazzaniga, M. S. (1992). Reading with a limited lexicon in the right hemisphere of a callosotomy patient. Neuropsychologia, 30, 187-200.

Beeman, M. J., \& Bowden, E. M. (2000). The right hemisphere maintains solution-related activation for yet-to-be-solved problems. Memory \& Cognition, 28, 1231-1241.

Beeman, M. J., Bowden, E. M., \& Gernsbacher, M. A. (2000). Right and left hemisphere cooperation for drawing predictive and coherence inferences during normal story comprehension. Brain and Language, 71, 310-336.

Beeman, M. J., \& Chiarello, C. (1998). Complementary right- and left-hemisphere language comprehension. Current Directions in Psychological Science, 7, 2-8.

Beeman, M., Friedman, R., Grafman, J., Perez, E., Diamond, S., \& Lindsay, M. (1994). Summation priming and coarse coding in the right hemisphere. Journal of Cognitive Neuroscience, 6, 26-45.

Blasi, V., Young, A. C., Tansy, A. P., Petersen, S. E., Snyder, A. Z., \& Corbetta, M. (2002). Word retrieval learning modulates right frontal cortex in patients with left frontal damage. Neuron, 36, 159-170.

Bookheimer, S. (2002). Functional MRI of language: New approaches to understanding the cortical organization of semantic processing. Annual Review of Neuroscience, 25, 151-188.

Brownell, H., Michel, D., Powelson, J., \& Gardner, H. (1983). Surprise but not coherence: Sensitivity to verbal humor in right-hemisphere patients. Brain and Language, 18, 20-27.

Buckner, R. L. (2003). Functional-anatomic correlates of control processes in memory. Journal of Neuroscience, 23, 3999-4004.

Buckner, R. L., Corbetta, M., Schatz, J., Raichle, M. E., \& Petersen, S. E. (1996). Preserved speech abilities and compensation following prefrontal damage. Proceedings of the National Academy of Sciences, U.S.A., 93, 1249-1253.

Burgess, C., \& Simpson, G. (1988). Cerebral hemispheric mechanisms in the retrieval of ambiguous word meaning. Brain and Language, 42, 203-217.

Calvert, G. A., Brammer, M. J., Morris, R. G., Williams, S. C., King, N., \& Matthews, P. M. (2000). Using fMRI to study recovery from acquired dysphasia. Brain and Language, 71, 391-399.

Chiarello, C., Burgess, C., Richards, L., \& Pollock, A. (1990). Semantic and associative priming in the cerebral hemisphere: Some words do, some words don't. . .sometimes, some places. Brain and Language, 38, 75-104.

Coney, J. (2002). The effect of associative strength on priming in the cerebral hemispheres. Brain and Cognition, 50, $234-241$.

Coulson, S. (2000). Semantic leaps: Frame-shifting and conceptual blending in meaning construction. Cambridge: Cambridge University Press.

Coulson, S., \& Kutas, M. (2001). Getting it: Human event-related brain response in good and poor comprehenders. Neuroscience Letters, 316, 71-74.

Coulson, S., \& Lovett, C. (2004). Handedness, hemispheric asymmetries, and joke comprehension. Cognitive Brain Research, 19, 275-288.

Delis, D., Robertson, L. C., \& Efron, R. (1986). Hemispheric specialization of memory for visual hierarchical stimuli. Neuropsychologia, 24, 205-214.

Duncan, J., \& Owen, A. (2000). Common regions of the human frontal lobe recruited by diverse cognitive demands. Trends in Neurosciences, 23, 475-483.

Faust, M., \& Gernsbacher, M. (1996). Cerebral mechanisms for suppression of inappropriate information during sentence comprehension. Brain and Language, 53, $234-259$.

Federmeier, K. D., \& Kutas, M. (1999). Right words and left words: Electrophysiological evidence for hemispheric differences in meaning processing. Cognitive Brain Research, 8, 373-392.

Federmeier, K. D., \& Kutas, M. (2002). Picture the difference: Electrophysiological investigations of picture processing in the two cerebral hemispheres. Neuropsychologia, 40, $730-747$.

Fink, G., Halligan, P. W., Marshall, J. C., Frith, C. D., Frackowiak, R., \& Dolan, R. J. (1996). Where in the brain does visual attention select the forest and the trees? Nature, 382, 626-628.

Galuske, R. A., Schlote, W., Bratzke, H., \& Singer, W. (2000). Interhemispheric asymmetries of the modular structure in human temporal cortex. Science, 289, 1946-1949.

Gazzaniga, M. S., \& Sperry, R. W. (1967). Language after section of the cerebral commissures. Brain, 90, 131-138.

Goel, V., \& Dolan, R. J. (2001). The functional anatomy of humor: Segregating cognitive and affective components. Nature Neuroscience, 4, 237-238.

Hillyard, S., \& Anllo-Vento, L. (1998). Event-related brain potentials in the study of visual selective attention. Proceedings of the National Academy of Sciences, U.S.A., 95, 781-787.

Hough, M. S. (1990). Narrative comprehension in adults with right and left hemisphere brain-damage: Theme organization. Brain and Language, 38, 253-277. 
Hutsler, J. G., \& Gazzaniga, M. S. (1995). Hemispheric differences in the density of parvalbumin-containing interneurons are found within language-associated regions of the human cerebral cortex. Journal of Cognitive Neuroscience, Supplement.

Hutsler, J., \& Galuske, R. A. (2003). Hemispheric asymmetries in cerebral cortical networks. Trends in Neurosciences, 26, $429-435$.

Huynh, H., \& Feldt, L. S. (1978). Estimation of the box correction for degrees of freedom from sample data in the randomized block and split plot designs. Journal of Educational Statistics, 1, 69-82.

Jacobs, B., Batal, H. A., Lynch, B., Ojemann, G., Ojemann, L. M., \& Scheibel, A. B. (1993). Quantitative dendritic and spine analyses of speech cortices: A case study. Brain and Language, 44, 239-253.

Jacobs, B., Schall, M., \& Scheibel, A. B. (1993). A quantitative dendritic analysis of Wernicke's area in humans. II. Gender, hemispheric, and environmental factors. Journal of Comparative Neurology, 327, 97-111.

Kutas, M., Federmeier, K. D., Coulson, S., King, J., \& Munte, T. F. (2000). Language. In J. T. Cacioppo, L. G. Tassinary \& G. Berntson (Eds.), Handbook of psychophysiology (2nd ed., pp. xiii, 1039). Cambridge: Cambridge University Press.

Kutas, M., Van Petten, C., \& Besson, M. (1988). Event-related potential asymmetries during the reading of sentences. Electroencephalography and Clinical Neurophysiology, 69, $218-233$.

Nuwer, M., Comi, G., Emerson, R., Fuglsang-Frederiksen, A., Guerit, J.-M., Hinrichs, H., \& Rappelsburger, P. (1998). IFCN standards for digital recording of clinical EEG. Electroencephalography and Clinical Neurophysiology, 106, 259-261.

Oldfield, R. C. (1971). The assessment and analysis of handedness: The Edinburgh Inventory. Neuropsychologia, 9, 97-113.

Osterhout, L., \& Holcomb, P. (1995). Event related potentials and language comprehension. In M. D. Rugg, M. G. H. Coles (Eds.), Electrophysiology of mind: Event-related brain potentials and cognition (pp. 171-215). Oxford University Press.
Roskies, A., Fiez, J., Balota, D., Raichle, M. E., \& Petersen, S. E. (2001). Task-dependent modulation of regions in the left inferior frontal cortex during semantic processing. Journal of Cognitive Neuroscience, 13, 829-843.

Shammi, P., \& Stuss, D. T. (1999). Humour appreciation: A role of the right frontal lobe. Brain, 122, 657-666.

St. George, M., Kutas, M., Martinez, A., \& Sereno, M. I. (1999). Semantic integration in reading: Engagement of the right hemisphere during discourse processing. Brain, 122, $1317-1325$.

Staudt, M., Lidzba, K., Grodd, W., Wildgruber, D., Erb, M., \& Krageloh-Mann, I. (2002). Right-hemispheric organization of language following early left-sided brain lesions: Functional MRI topography. Neuroimage, 16, $954-967$.

Stemmer, B. (1994). A pragmatic approach to neurolinguistics: Requests (re)considered. Brain and Language, 46, 565-591.

Stemmer, B., Giroux, F., \& Joanette, Y. (1994). Production and evaluation of requests by right hemisphere brain-damaged individuals. Brain and Language, 47, 1-31.

Van Kleek, M. (1989). Hemispheric differences in global versus local processing of hierarchical visual stimuli by normal subjects: New data and a meta-analysis of previous studies. Neuropsychologia, 27, 1165-1178.

Van Lancker, D. R., \& Kempler, D. (1987). Comprehension of familiar phrases by left- but not by right-hemisphere damaged patients. Brain and Language, 32, 265-277.

Van Petten, C. (1995). Words and sentences: Event-related brain potential measures. Psychophysiology, 32, 511-525.

Wagner, A., Pare-Blagoev, E., Clark, J., \& Poldrack, R. (2001). Recovering meaning: Left prefrontal cortex guides controlled semantic retrieval. Neuron, 31, 329-338.

Yamaguchi, S., Yamagata, S., \& Kobayashi, S. (2000). Cerebral asymmetry of the "top-down" allocation of attention to global and local features. Journal of Neuroscience, 20, RC72.

Zaidel, E. (1990). Language functions in the two hemispheres following complete cerebral commissurotomy and hemispherectomy. In F. Boller \& G. Grafman (Eds.), Handbook of neuropsychology (vol. 4, pp. 115-150) Amsterdam: Elsevier. 